\title{
Research Advances in Pick’s Disease: A New Biomarker Candidate
}

\section{Wenhui $\mathrm{Hu}^{1}$, and Jin Jun Luo ${ }^{2 *}$ \\ ${ }^{1}$ Department of Neuroscience, Temple University School of Medicine, Philadelphia, USA}

${ }^{2}$ Departments of Neurology and Pharmacology, Temple University School of Medicine, Philadelphia, USA

The eponym Pick's disease (PiD) was named after Arnold Pick, a German neurologist, who first described this rare neurodegenerative disease in 1892 [1]. Current knowledge indicates that PiD is a member of a group of heterogeneous neurodegenerative disorders with similar or related histopathologic and clinical features known as frontotemporal lobal degeneration or dementia (FTD) [2,3]. FTD may also be called Pick complex [3] including PiD, primary progressive aphasia, semantic dementia, FTD with parkinsonism (FTDP) linked with chromosome-17, FTD with amyotrophic lateral sclerosis (FTDALS) linked to a newly identified gene named as C9ORF72 in the chromosome 9 open reading frame 72 [4], corticobasal degeneration (CBD) [5], and others [2,3]. FTD is the first or second most common cause of dementia among individuals younger than 60 years and the fourth most common cause of dementia in the USA. It has been noted that the incidence rates of FTD in 100,000 were 2.2 for ages 40 to 49 , 3.3 for ages 50 to 59 , and 8.9 for ages 60 to 69 [6], and range between $4 \%$ and $20 \%$ within dementia and $12 \%$ before the age of 65 [7]. Autopsy findings showed the occurrence between $2 \%$ and $20 \%$ [7-9]. Prevalence was estimated as 15 per 100,000 (8.4 to 27.0) in the 45- to 64-year-old population [10,11]. PiD affects in individuals between the ages of 40 and 60 years old but can be seen individuals between 20 to 80 years old. A striking male preponderance (14:3) has been noted [11].

Pathologically, PiD is defined on the basis of histological appearance of neuronal loss and gliosis in severely circumscribed atrophy involving the anterior temporal and frontal lobes, the orbital frontal and the medial temporal lobes but sparing the posterior part of the superior temporal, the pre- and postcentral gyri, and parietal and occipital lobes [12]. The atrophy is usually worse on the dominant hemisphere. The ventricles, especially the frontal and temporal horns of the lateral ventricles, are enlarged. Pick cells and Pick bodies (PB), first described by Alois Alzheimer in 1911, are pathognomonic markers for PiD [13]. Pick cells are large, swollen, ballooned, diffusely argyrophilic neurons with vacuolated cytoplasm. They are common in the zones between severely affected and relatively preserved regions of the cortex and are also present in the cingulated gyrus, insular cortex, claustrum and occasionally in the anterior portion of the neostriatum. $\mathrm{PB}$ are argyrophilic intracellular spherical inclusions containing abnormal tau fibrils $[14,15]$ arranged in a disorderly array. The major structural component of phosphorylated neurofilaments in $\mathrm{PB}$ is the microtubule-associated protein tau. In physiologic conditions, tau proteins are phosphorylated and assembled into filaments [16] exerting a fundamental role in axonal transport [17]. Abnormally phosphorylated and aggregated tau proteins are biochemical markers of various forms of neurodegenerative dementias, including Alzheimer's disease $(\mathrm{AD}), \mathrm{PiD}, \mathrm{CBD}$, progressive supranuclear palsy (PSP), and FTDP, collectively called tauopathies $[14,18]$. PB are mostly located in the layers II, III and IV of the neocortex $[19,20]$. PB can be detected with various antibodies including those to tau, ubiquitin, alphasynuclein, apolipoprotein E, N-terminal amyloid precursor protein, Alz-50, clathrin, synaptophysin, neuronal surface glycoside, and $\beta \mathrm{II}$ tubulin. Antibodies to abnormally phosphorylated tau proteins are the best markers for PB and Pick cells [21]. Additionally, silver stains can be useful in distinguishing PiD from AD [22].
The etiological role of tau proteins in neurodegeneration has been studied through the identification of mutations in Tau in the familial form of dementia and/or parkinsonism [23,24]. The gene that encodes tau is on chromosome 17q21. In an adult human brain there are six tau isoforms. Alternative splicing of exons 2, 3, and 10 within the tau gene produces 6 isoforms that create 3 or 4 repeats of the microtubule binding domain of tau. Altering this ratio of 4-repeats to 3-repeats results in various pathologies. For example, AD exhibits 6 tau isoforms, 4-repeat tau is more common in CBD and PSP, whereas initial studies showed PiD has only 3-repeat isoforms [25] but, subsequent studies showed equal frequency of 4-repeat to 3-repeat tau in PiD [26].

Based on the immunocytochemical findings, FTD may be further classified into three subtypes [12,27]. Type A is the classic $\mathrm{PiD}$, characterized by front temporal and limbic degeneration with PB and Pick cells. Type B has superior frontal and parietal atrophy with Pick cells but no PB. It can be seen in CBD or FTDP with asymmetrical corticospinal and extra pyramidal symptoms and signs, and degeneration of the cortex, substantia nigra, striatum, and other deep nuclei. Type $\mathrm{C}$ is characterized by the absence of both $\mathrm{PB}$ and Pick cells with either circumscribed or diffuse cortical atrophy with varying involvement of deep nuclei $[12,19]$.

Clinically, there are two core PiD symptomatic patterns involving behavior and language. Features of behavioral changes can be either impulsive (disinhibited) or bored (apathetic) and include inappropriate social behavior; lack of social tact or empathy; loss of insight into the behaviors of oneself and others; an increased interest in sex; changes in food preferences; agitation or, conversely, blunted emotions; neglect of personal hygiene; repetitive or compulsive behavior, and decreased energy and motivation. Features of language disturbance include difficulty in thinking or understanding speech, often in conjunction with abnormal behavioral symptoms but with intact spatial skills and memory $[3,14,15]$.

On neurologic examination, PiD patients typically show frontal release signs of primitive, such as grasp and palmomental, reflexes. Extrapyramidal features such as rigidity, gait instability, a masked face, and micrographia are frequently encountered but a resting tremor is rare. Fasciculation, muscle wasting, motor weakness, swallowing difficulty with an attenuated gag reflex and effortful speech may be seen $[3,14,15]$. Signs of ocular and cerebellar dysfunctions such as ataxia,

*Corresponding author: Jin Jun Luo, Temple University School of Medicine, 3401 North Broad Street, Philadelphia, USA, E-mail: jluo@temple.edu

Received December 11, 2012; Accepted December 18, 2012; Published December 20, 2012

Citation: Hu W, Luo JJ (2013) Research Advances in Pick's Disease: A New Biomarker Candidate. J Neurol Neurophysiol 4:e112. doi:10.4172/21559562.1000e112

Copyright: (c) $2013 \mathrm{Hu} \mathrm{W}$, et al. This is an open-access article distributed under the terms of the Creative Commons Attribution License, which permits unrestricted use, distribution, and reproduction in any medium, provided the original author and source are credited. 
dysmetria, and sensory defects are not typically associated with PiD. Because of no specific neurologic signs for the diagnosis of PiD, cognitive and behavioral tests are usually applied. Detailed neuropsychologic test batteries have been developed to help, but many of these comparative studies have limitations, in distinguishing dementia between PiD and $\mathrm{AD}$ [28]. The Mini Mental State Examination (MMSE) is not a sensitive tool for screening patients with $\mathrm{PiD}$, since profoundly impaired $\mathrm{PiD}$ patients can have a normal MMSE [29]. Atrophy of front and temporal lobes is demonstrated by neuroimaging such as brain CT or MRI. The lobar hypoperfusion is displayed by functional neuroimaging such as single-photon emission computed tomography. However, the gold standard for the diagnosis of FTD is neuropathology.

There is no specific treatment for PiD. No clinical trials have been conducted. Empirically treating PiD patients with cholinesterase inhibitors, antidepressants, selective serotonin reuptake inhibitors, and antipsychotics showed no satisfactory effects. PiD progresses steadily and often rapidly, ranging from less than 2 years to more than 10 years, with an average approximately 6 years [30]. Notably, PiD patients with main symptoms of speech and language disturbance (primary progressive aphasia) may survive 5 years longer than those with behavioral symptoms (behavioral variant) [31], and may preserve the ability to function at home for 10 or more years after onset.

The cause of PiD is unknown. Familial forms of Pick-complex dementias has been found to be linked to chromosome 17,9 , and 3 [3]. The familial form is approximately $30-40 \%$ of all FTD cases but tau mutation has been found only in approximate $10 \%$ of the families tested, and not in any sporadic cases [10]. In addition to the tau mutation, TDP-43 gene mutation is identified in FTDP and FTDALS [2,32]. TDP-43 is a major component of inclusions in ALS. In transgenic mice TDP-43 causes spinal and frontal neurodegeneration without protein aggregations, indicating that the mechanisms for the development of the disease is likely due to altered function of the DNA/ RNA binding rather than a toxic effect of the protein [33].

In a recently published issue in the Journal of Neurology and neurophysiology, Nishijima et al. [34] reported a possible biomarker candidate named calmodulin-like skin protein (CLSP) for PiD. They identified CLSP from a solubilized PB sample using a lasermicrodissection system and Unfoldin-modified proteomic analysis. Unfoldin, named by the authors, was purified from yeast cells consisting of a homo-oligomer of 10-12 subunits, each of which is identical to Aip2p/Dld2p from YDL178w yeast. The authors found that Unfoldin can effectively solubilize aggregated proteins including $\mathrm{PB}$ in $\mathrm{PiD}$ [35]. Therefore, Unfoldin-solubilized samples can be used for many downstream analyses such as immunoblotting, immunoprecipitation, protein sequencing, etc. While their study advances our understanding of $\mathrm{PB}$ in $\mathrm{PiD}$, caution is warranted in interpreting the results. As a novel biomarker candidate for PiD, CLSP needs further confirmation. Studies using immunofluorescent double staining and confocal imaging analysis in tissue sections may be helpful to demonstrate the colocalization of CLSP and abnormally phosphorylated tau protein in PiD. Because extracellular PB may lose tau immunoreactivity due to proteolysis [36], the possibility of changes in PB antigenicity after Unfoldin-treatment should be tested. It would be interesting to know whether CLSP is expressed specifically or nonspecifically in PB, for example, chromogranin A staining of $\mathrm{PB}$ can be observed in other neurodegenerative disorders [37].

Dementia is a natural dilemma in a human's life. Advanced technology with scrutinized clinical and laboratory research would help shed more light on the pathogenesis of PiD and to establish a strategy applicable to early diagnosis, effective treatment and, possibly, prevention of $\mathrm{PiD}$ in the future.

\section{References}

1. Pick A (1892) Über die Beziehungen der senilen Hirnatrophie zue Aphasie [On the relationship between senile cerebral atrophy and aphasia. Prager Medicinische Wochenschrift 17: 165-167.

2. Takeda N, Kishimoto Y, Yokota O (2012) Pick's disease. Adv Exp Med Biol 724: 300-316

3. Kertesz A (2006) Progress in clinical neurosciences: Frontotemporal dementiapick's disease. Can J Neurol Sci 33: 141-148.

4. Renton AE, Majounie E, Waite A, Simon-Sanchez J, Rollinson S, et al. A hexanucleotide repeat expansion in C9ORF72 is the cause of chromosome 9p21-linked ALS-FTD. Neuron 72: 257-268.

5. Reich SG, Grill SE (2009) Corticobasal degeneration. Curr Treat Options Neurol 11: 179-185.

6. Knopman DS, Petersen RC, Edland SD, Cha RH, Rocca WA (2004). The incidence of frontotemporal lobar degeneration in Rochester, Minnesota, 1990 through 1994. Neurology 62: 506-508

7. Nowacki P, Zdziarska B, Zyluk B, Jankowska-Kurek I (1993) Hemiballismus in patient with blastic phase of chronic myelogenous leukemia. Neuropatol Pol 31: 143-148.

8. Knopman DS, Mastri AR, Frey WH 2nd, Sung JH, Rustan T (1990) Dementia lacking distinctive histologic features: a common non-Alzheimer degenerative dementia. Neurology 40: 251-256.

9. Klatka LA, Schiffer RB, Powers JM, Kazee AM (1996) Incorrect diagnosis of Alzheimer's disease. A clinicopathologic study. Arch Neurol 53: 35-42.

10. Bird T, Knopman D, VanSwieten J, Rosso S, Feldman H, et al (2003) Epidemiology and genetics of frontotemporal dementia/Pick's disease. Ann Neurol 54: S29-S31.

11. Ratnavalli E, Brayne C, Dawson K, Hodges JR (2002) The prevalence of frontotemporal dementia. Neurology 58: 1615-1621.

12. Dickson DW (2001) Neuropathology of Pick's disease. Neurology 56: S16-S20

13. Jellinger KA (1995) Neuropathological criteria for Pick disease and frontotemporal lobe dementia. In: Cruz-Sanchez, ed. Neuropathological Diagnostic Criteria. Amsterdam: IOS Press: 35-54.

14. Graff-Radford NR, Woodruff BK (2007) Frontotemporal dementia. Semin Neurol 27: 48-57.

15. Forman MS, Farmer J, Johnson JK, Clark CM, Arnold SE, et al. (2006) Frontotemporal dementia: clinicopathological correlations. Ann Neurol 59: 952 962.

16. Lee VM, Goedert M, Trojanowski JQ (2001) Neurodegenerative tauopathies Annu Rev Neurosci 24: 1121-1159.

17. van Swieten J, Spillantini MG (2007) Hereditary frontotemporal dementia caused by Tau gene mutations. Brain Pathol 17: 63-73.

18. Goedert M, Jakes R (2005) Mutations causing neurodegenerative tauopathies. Biochim Biophys Acta. 1739: 240-250.

19. (2000) Case records of the Massachusetts General Hospital. Weekly clinicopathological exercises. Case 11-2000. A 74-year-old man with memory loss, language impairment, and personality changes. N Engl J Med 342: 11101117.

20. Wang LN, Zhu MW, Feng YQ, Wang JH (2006) Pick's disease with Pick bodies combined with progressive supranuclear palsy without tuft-shaped astrocytes: a clinical, neuroradiologic and pathological study of an autopsied case. Neuropathology 26: 222-230.

21. Armstrong RA, Cairns NJ, Lantos PL (1999) Quantification of pathologica lesions in the frontal and temporal lobe of ten patients diagnosed with Pick's disease. Acta Neuropathol 97: 456-462.

22. Uchihara T, Tsuchiya K, Nakamura A, Akiyama H (2005) Silver staining profiles distinguish Pick bodies from neurofibrillary tangles of Alzheime type: comparison between Gallyas and Campbell-Switzer methods. Acta Neuropathol 109: 483-489.

23. Hutton M, Lendon CL, Rizzu P, Baker M, Froelich S, et al. (1998) Association of 
Citation: Hu W, Luo JJ (2013) Research Advances in Pick's Disease: A New Biomarker Candidate. J Neurol Neurophysiol 4:e112. doi:10.4172/2155$9562.1000 \mathrm{e} 112$

missense and 5'-splice-site mutations in tau with the inherited dementia FTDP17. Nature 393: 702-705

24. Spillantini MG, Murrell JR, Goedert M, Farlow MR, Klug A, et al. (1998) Mutation in the tau gene in familial multiple system tauopathy with presenile dementia. Proc Natl Acad Sci U S A 95: 7737-7741.

25. Buée L, Delacourte A (1999) Comparative biochemistry of tau in progressive supranuclear palsy, corticobasal degeneration, FTDP-17 and Pick's disease. Brain Pathol 9: 681-693.

26. Zhukareva V, Mann D, Pickering-Brown S, Uryu K, Shuck T, et al. (2002) Sporadic Pick's disease: a tauopathy characterized by a spectrum of pathological tau isoforms in gray and white matter. Ann Neurol 51: 730-739.

27. Constantinidis J (1985) Pick dementia. Anatomoclinical correlations and pathophysiological considerations. Karger: Basel Switzerland.

28. Cummings JL, Mega M, Gray K, Rosenberg-Thompson S, Carusi DA, et al. (1994) The Neuropsychiatric Inventory: comprehensive assessment of psychopathology in dementia. Neurology 44: 2308-2314.

29. Gregory CA, Serra-Mestres J, Hodges JR (1999) Early diagnosis of the frontal variant of frontotemporal dementia: how sensitive are standard neuroimaging and neuropsychologic tests? Neuropsychiatry Neuropsychol Behav Neurol 12: 128-135.

30. Hodges JR, Davies R, Xuereb J, Kril J, Halliday G (2003) Survival in frontotemporal dementia. Neurology 61: 349-354.
31. Piguet O, Halliday GM, Reid WG, Casey B, Carman R, et al. (2011) Clinical phenotypes in autopsy-confirmed Pick disease. Neurology 76: 253-259.

32. Rohrer JD, Geser F, Zhou J, Gennatas ED, Sidhu M, et al. (2010) TDP-43 subtypes are associated with distinct atrophy patterns in frontotemporal dementia. Neurology 75: 2204-2211.

33. Wegorzewska I, Bell S, Cairns NJ, Miller TM, Baloh RH (2009) TDP-43 mutant transgenic mice develop features of ALS and frontotemporal lobar degeneration. Proc Natl Acad Sci U S A 106: 18809-18814.

34. Nishijima K, O'hara K, Kaneko K, Hachiya N. Calmodulin-Like Skin Protein (CLSP) is a Novel Biomarker Candidate for Pick's Disease by Unfoldin-Modified Proteomic Analysis. J Neurol Neurophysiol 2012:S11-003.

35. Hachiya NS, Kaneko K (2007) Investigation of laser-microdissected inclusion bodies. Methods Cell Biol 82: 355-375.

36. Izumiyama Y, Ikeda K, Oyanagi S (1994) Extracellular or ghost Pick bodies and their lack of tau immunoreactivity: a histological, immunohistochemical and electron microscopic study. Acta Neuropathol 87: 277-283.

37. Yasuhara O, Kawamata T, Aimi Y, McGeer EG, McGeer PL (1994) Expression of chromogranin $A$ in lesions in the central nervous system from patients with neurological diseases. Neurosci Lett: 13-16. 